\title{
La Loi et les lois
}

Pierre Moor

\section{(2) OpenEdition}

Journals

Édition électronique

URL : http://journals.openedition.org/ress/464

DOI : $10.4000 /$ ress.464

ISSN : 1663-4446

\section{Éditeur}

Librairie Droz

\section{Édition imprimée}

Date de publication : 1 janvier 2006

Pagination : 89-102

ISBN : 2-600-00959-0

ISSN : 0048-8046

\section{Référence électronique}

Pierre Moor, «La Loi et les lois », Revue européenne des sciences sociales [En ligne], XLIV-133 | 2006, mis en ligne le 10 novembre 2009, consulté le 21 décembre 2020. URL : http:// journals.openedition.org/ress/464 ; DOI : https://doi.org/10.4000/ress.464 


\title{
Pierre MOOR
}

\section{LA LOI ET LES LOIS}

\section{L'HOMME DE LA CAMPAGNE, OU LE MESSAGE RETENU}

\subsection{Ainsi Kafka commence-t-il sa nouvelle Devant la loi ${ }^{1}$ :}

\begin{abstract}
«Devant la loi se dresse le gardien de la porte. Un homme de la campagne se présente et demande à entrer dans la loi. Mais le gardien dit que pour l'instant il ne peut pas lui accorder l'entrée. L'homme réfléchit, puis demande s'il lui sera permis d'entrer plus tard. "C'est possible", dit le gardien, "mais pas maintenant".»
\end{abstract}

Dure ensuite l'attente de toute une vie. Au moment de mourir, l'homme demande une dernière fois :

\begin{abstract}
«"Si chacun aspire à la loi, comment se fait-il que durant toutes ces années personne autre que moi n'ait demandé à entrer?" Le gardien de la porte, sentant venir la fin de l'homme, lui rugit à l'oreille pour mieux atteindre son tympan presque inerte: "Ici nul autre que toi ne pouvait pénétrer, car cette entrée n'était faite que pour toi. Maintenant, je m’en vais et je ferme la porte".»
\end{abstract}

Dans la parabole, la Loi (la majuscule en rend mieux compte) est norme pure ${ }^{2}$ : elle équivaut à une interdiction absolue d'accès, non pas parce que la présence du gardien rend en fait impossible d'y pénétrer, mais parce que, se présentant privée de tout contenu, elle est impossible à respecter. Elle est la Loi en elle-même, on devrait dire la Loi de la Loi : ce qui, une fois toute norme dépouillée de ce qui lui est propre, subsiste pour toutes comme normativité pure. Ainsi, tout sens étant évacué, elle est Parole vide - un oxymoron.

L'homme n'a - de son vivant - c'est-à-dire en tant qu'il est ce qu'il est - rien pour la comprendre, donc rien à y comprendre. Bien plus: le gardien a attendu sa mort pour lui faire sa communication: la Loi n'a (plus) rien à lui dire.

Il n'y a par conséquent pas de règle - sauf dans la tête du gardien, qui la «garde» pour lui; et cette règle retenue jusqu'au moment ultime est, dans la seule

On notera que le traducteur a mis une minuscule à «loi», ce à quoi il n'était pas obligé, puisque, en allemand, les noms même communs portent toujours une majuscule. «Devant la loi », in La métamorphose et autres récits, trad. Alexandre Vialatte (1938), Paris, Gallimard, 1985, p. 153 ss; la même parabole est reprise dans Le Procès, trad. Alexandre Vialatte (1933), Paris, Gallimard (Folio), 1978 , p. 308 ss.

2 Quasiment dans le sens où, à la même époque, Kelsen concevait une Théorie pure du droit comme système formel d'habilitations. 
chose qu'elle dit lorsqu'il n'y a plus rien à dire, individuelle («cette porte était la tienne»). En réalité, c'est cela qu'il garde (et non pas l'accès), pour ne le communiquer que lorsque cette communication sera inutile; il ne le communique que lorsque la communication est privée de sens puisqu'elle n'apporte rien qui puisse être retravaillé par l'homme dans un retour de communication. La communication est, au moment où elle est faite, et du fait du moment même où elle est faite, immédiatement une non-communication ${ }^{3}$.

On retrouve une situation analogue dans la Colonie pénitentiaire ${ }^{4}$ : les condamnés à mort reçoivent communication du jugement au moment où la machine même qui les exécute le grave sur leur peau.

Et pourtant, le gardien le dit expressément, «cette entrée était faite pour toi.» L'homme, lui, s'était imaginé les choses différemment: «La loi ne doit-elle pas être accessible à tous et toujours?». Mais non: il n'y a pas une même loi pour tous. S'il n'y a pas une même loi pour tous, la seule loi commune à tous est que chacun est sous l'empire de sa propre loi: ce serait là la Loi de la Loi, cette Parole qui s'adresse individuellement à chacun mais se répète identiquement pour tous horrible psittacisme - pour ne dire qu'une chose et ne jamais permettre de comprendre quoi que ce soit d'autre sur cette seule chose qu'elle dit: «Je suis ta Loi ». Elle ne s'incarne jamais en signes parce qu'elle est immédiatement singulière et que, dès lors, son universalité n'est que purement formelle: elle est universellement immédiatement singulière.

1.2. Quel est le monde de cette Loi? Un monde où il n'y a rien à interpréter, donc rien à répondre, ou bien où toute interprétation est possible et n'est par conséquent rien d'autre que n'importe quoi. Mais, s'il n'y a rien à répondre, la conséquence n'en est pas qu'il n'y a rien non plus de quoi répondre - sinon ce ne serait pas la Loi: bien au contraire, il y a à répondre de $t^{\prime} u t^{5}$. Un monde où tous sont par avance condamnés parce qu'ils sont ce qu'ils sont ${ }^{6}$, quoi qu'ils disent et quoi qu'ils fassent - à moins (Calvin!) de la grâce divine - laquelle est d'ailleurs tout autant Parole pure et nue que la condamnation elle-même. A chacun s'adresse cette Parole qui est la Loi et qui est personnellement destinée - prédestinée - à chacun. Chez Kafka, sans réponse possible; chez Calvin, avec au contraire une réponse humaine possible - celle de la foi, également parole pure, sans aucun

3 François Ost, Raconter la loi - Aux sources de l'imaginaire juridique, Paris, Odile Jacob, 2004, p. 346, décrivant l'univers des héros kafkaïens: «un monde d'implacables commandements totalement dépourvus de sens et de valeur positive (libératrice) pour leurs destinataires ». Le présent texte doit neaucoup à cet ouvrage: que son auteur en soit remercié.

${ }^{4}$ La colonie pénitentiaire. Nouvelles suivies d' un Journal intime, trad. Jean Starobinski, Paris, Egloff, 1945, p. 71 ss.

5 Pour reprendre Ost, p. 344, et, sur le passage de la culpabilité à l'autoaccusation, p. 361, en jouant sur deux sens possibles de «répondre». Disons dès maintenant que, dans le monde des lois mené dans toute sa logique, vaut au contraire la présomption d'innocence: Bjarne Melkevik, Considérations juridico-philosophiques, Québec, Les Presses de l’Université Laval, 2005, p. 58 ss (précisément en rapport avec Devant la loi et le Procès).

6 Ost remarque qu'il y a inversion de la succession temporelle de la règle et de la sanction: d'abord intervient la sanction («tu mourras») puis est énoncée la règle («il t'est interdit») (p. 348). Il en découle une inversion logique: «Tu es mortel, donc il t'est interdit». 
contenu autre que d'être une réponse à ce Dieu qui accordera en échange - sa grâce.

Disons-le dès maintenant: cette Loi est tout autre chose donc que la loi - sans majuscule! Celle-ci aurait-elle été inventée pour apprivoiser la Loi? Y réussitelle? Comment? Ce sont là les questions que je veux aborder.

Une voie pourrait nous y mener: si l'homme ne veut pas attendre une grâce que rien ne lui assure, il doit prendre sur lui de transgresser. Mais qu'arriverait-il s'il prenait sur lui de ne pas se plier à la Loi? Kafka ne répond jamais à cette question - K. se rend devant le juge, dont il attend la Justice - mais il ne sait pas $a u$ monde laquelle - il s'y rend quand même.

Qu'est-ce que cela pourrait être que transgresser? La transgression paraît proprement impensable, puisqu'il n'y a pas de norme qui pose une règle.

Peut-on tracer une analogie avec la Parole - également pure et nue - du Père ${ }^{7}$ ? Que la psychanalyse apprend à apprivoiser, en lui donnant, par le travail sur le souvenir, figure humaine - le restitue donc comme un homme ayant communiqué quelque chose à interpréter? La psychanalyse n'est-elle pas l'acte qui consiste à pénétrer quand même, c'est-à-dire à transgresser? Et pénétrer signifie transformer en langage une Parole qui prétendait être pure de tout signe, pour pouvoir y répondre de manière autonome.

Finalement, ne revient-elle pas à interpréter ce que (ne) dit (pas) le Père en Le privant, Lui, de son droit unique à la Parole - puisque cette Parole, primitivement figée dans ce qu'elle a laissé derrière elle, est dès lors (et uniquement dès lors) ouverte unilatéralement par le fils lui-même à l'interprétation? Interprétation qui en fait de simples paroles, des faits de langue. La Parole est transmutée en signes que le fils fait dire, dans le transfert, par l'analyste. Par cela même son pouvoir est transgressé puisque le fils se l'approprie. N'est-ce pas en même temps, pour reprendre le thème de la Loi, l'invention de la politique en tant que procédure qui permet à la société de se donner elle-même ses lois?

La volonté d'interprétation est alors création de la possibilité même d'un sens, c'est-à-dire une langue - ce n'est pas l'existence antérieure d'un sens qui crée la possibilité de l'interpréter. Transgresser n'est donc pas pénétrer de force (renverser le gardien!) dans la maison - ce serait simplement prendre la place du Père: c'est bien plutôt lui tourner le dos pour inventer autre chose - bâtir sa propre maison, avec tous les risques qu'une telle aventure implique, sans craindre d'utiliser pour cela des matériaux que la père a fournis. Transgresser, autrement dit, c'est produire, par son travail, du sens là où il n'y en avait pas.

C'est inventer la loi comme sens, et la faire travailler à la place de la Loi. C'est substituer le père au Père. Mais il y faut un psychanalyste sur la personne de qui le transfert de l'un à l'autre puisse s'opérer. En politique, il n'y évidemment pas de divan, ni personne assis derrière; mais nous verrons que d'autres différenciations de rôle sont introduites.

Précisément pour Kafka: Lettre au père, trad. Marthe Robert, Paris, Gallimard, 1995 - lettre qui, significativement, a été écrite pour se parler à lui-même, et non pas à son père. 


\section{LA CITÉ INTERDITE, OU LE MESSAGE REFUSÉ}

Personne n'est écouté apportant le message d'un mort: telle est la conclusion d'une autre parabole de Kafka, évoquée par le texte de Gérard Timsit ${ }^{8}$, qui oppose les deux histoires - la première, dans laquelle, selon lui, l'institution porteuse de sens est anonyme, la seconde, dans laquelle elle s'incarne dans un individu.

Ce n'est pas la seule différence. Dans la première, il n'y a pas de message, parce que son destinataire meurt au moment où il le reçoit - et recevoir un message signifie pouvoir en faire quelque chose, ce que l'homme de la campagne ne peut pas. Recevoir un message trop tard signifie que ce n'est (plus) un message.

Dans la seconde, c'est l'expéditeur qui est mort au moment où le message doit être délivré. Il n'y a donc pas de réponse possible, et, à nouveau, ce qui est transmis n'est dès lors (plus) un message.

Ce qui, dans cette seconde histoire, est bizarre, c'est que l'auteur du message n'est pas n'importe qui (par exemple un homme de la campagne): c'est l'Empereur. Donc, pourrait-on croire, le Pouvoir lui-même. Voilà donc que l'autorité suprême est tout autant frappée d'impuissance - parce que la charge est occupée par un mortel. Le destinataire du message, semble-t-il, n'avait pas lu Kantorowicz (ce qu'on ne peut évidemment lui reprocher!) ${ }^{9}$. Et certes, on peut supposer que l'Empereur avait envoyé un message privé et que, décédé, sa parole était frappée de caducité. Mais si on pouvait admettre que le message avait été impérial, et non pas personnel, son destinataire l'aurait-il reçu, parce qu'il serait parti de l'axiome que l'Empereur en tant que tel est toujours en vie? Pas davantage, semble-t-il.

En effet, Kafka écrit que la Ville impériale ne reçoit aucun message et surtout pas celui d'un mort: "Là personne ne pénètre, même pas avec le message d'un mort $\gg^{10}$. Et cela même si ce mort est - a été - l'homme le plus puissant du monde, l'Empereur lui-même. Transcrit dans les images de la première nouvelle, même si celui qui attend devant la porte avait été doté des plus grands pouvoirs, même s'il était entré (et la description que le gardien fait à l'homme de la campagne des obstacles qu'il lui faudrait franchir correspond à celle des difficultés que le messager impérial a à vaincre), jamais ce qu'il aurait eu à dire - voire sous forme d'une simple question - n'aurait été entendu. L'Empereur n'a pas plus droit à la parole que l'homme de la campagne: face à la Loi, il est aussi singulier que lui, et, comme lui, et l'eût-il voulu, sa mort même l'empêche définitivement de tenter de prendre d'assaut la cité interdite pour y imposer la réception de ce qu'il avait à dire.

${ }^{8}$ Cf. sa contribution dans ce volume.

9 Mais Kafka l'avait précédé, quand il oppose à la souveraineté impériale l'empereur «actuel », dont aucune nouvelle ne peut parvenir utilement à ses sujets, tant ceux-ci en sont éloignés (dans la «Construction de la muraille de Chine», in: La colonie pénitentiaire, cit., p. 215); cette nouvelle contient d'ailleurs aussi la parabole du message impérial (p. 217 s.).

10 «Un message impérial», in: La métamorphose (cit.), p. 174. «Personne ne peut passer par là, et même avec le message d'un mort», dans la traduction de Starobinski. 


\section{LA NOBLESSE PERVERSE, OU LE MESSAGE ARBITRAIRE}

Il existe un très court texte de Kafka intitulé Au sujet des lois $^{11}$, qui traite aussi du monde normatif et de sa communication. Les lois qui nous régissent nous sont inconnues, elles sont le secret de la noblesse qui nous domine. A vrai dire, même si on ne peut que la présumer, une tradition bien établie veut qu'elles existent. Le seul moyen de les respecter est d'imiter les comportements de la noblesse. Mais interpréter ces comportements est une tâche infinie: il faut comprendre une longue tradition, consistant en une infinité de faits. Dans le quotidien, on en est réduit à présumer de vagues lignes directrices, peu sûres, de telle sorte qu'il n'est pas possible d'être certain que l'on se conforme effectivement à la loi alors même que l'on règle ses actes sur ceux qui la connaissent; le monde normatif se manifeste apparemment de manière incohérente, tant que, du moins, on n'aura pas suffisamment étudié ce qui nous est accessible - ce qui peut prendre des siècles. C'est comme si, dans la caverne platonicienne, les ombres étaient dotées d'une volonté propre qui leur permettrait de se déformer pour ne pas être ce qu'elles devraient être: les ombres des Idées ${ }^{12}$.

Il y a certes dans cette cité un petit parti révolutionnaire, qui ne voit dans le comportement de la noblesse que des actes arbitraires et rejette la tradition populaire. Mais il faudrait la renverser. Cependant, qui voudrait aller jusqu'à cette extrémité? Car «la seule Loi visible et indubitable qui nous est imposée est la noblesse, et nous voudrions encore nous priver de cette unique Loi? $\gg^{13}$

On retrouve ici, donc, la Loi, mais on a fait un pas, sans être cependant beaucoup plus avancé: elles ont bien un contenu, mais celui-ci reste non communiqué. Ou plutôt l'institution qui pourrait le faire - même le devrait, selon son statut, puisqu'elle constitue la noblesse - le fait de telle manière que les messages envoyés sont incertains, rendant inconnaissable le contenu impératif des lois. La Loi reprend donc sa majuscule. Elle ne descend pas dans le monde humain directement sous forme de signes. Les signes par lesquels son contenu est transmis sont indirects: ce sont les comportements de la noblesse, lesquels ne peuvent être perçus que comme aléatoires et sont donc indécodables: ils ne sont pas institutionnels, puisqu'ils dépendent de l'arbitraire de leur auteur. Par conséquent, comme dans Devant la Loi, comme dans Un message impérial, mais cette fois-ci non pas du fait du destinateur ni du destinataire, mais en raison du message luimême, dans lequel il n'y a en réalité rien à interpréter parce qu'on peut y lire tout et son contraire; autrement dit, l'interprétation ne peut avoir de fin et est condamnée à tourner à vide ${ }^{14}$.

${ }^{11}$ «Zur Frage der Gesetze», in: Meistererzählungen, Francfort s./M., Fischer, 1970, p. 360-362.

12 Il en va de même de tous les intermédiaires et satellites de la justice dans le Procès: Ost, p. 388 ss. Voir aussi dans le texte intitulé Le substitut: «[Les juges vénaux du Conseil de discipline] mentent comme seuls les juges savent le faire», et il ne se trouvera aucun juge intègre à qui on puisse confier la tâche de les juger (CEuvres complètes, t. 2, p. 348 s.).

13 Traduction de François Ost (p. 385).

${ }^{14}$ C'est la situation des livres sacrés en tant que message de la parole divine: dès lors qu'un canon en fixe définitivement le texte, il devient impossible d'obtenir une réponse aux questions que leur interprétation provoque (ne serait-ce que pour obtenir confirmation de son exactitude), si ce n'est par 


\section{PROBLÈMES DE MESSAGERIE}

4.1. Il y a des problèmes que nous appellerons familièrement de messagerie. Le message est émis trop tard par son destinateur, ou le destinataire refuse de le recevoir, ou il est rendu confus par ses intermédiaires. Qu'est-ce qui ne fonctionne pas?

Faisons l'hypothèse que la source de ces problèmes réside dans le fait que, dans chacune de ces trois situations, le message est à la libre disposition de celui dont la position lui permet de le maîtriser. Le destinateur le retient, le destinataire le refuse, l'intermédiaire le brouille, sans que les deux autres parties puissent intervenir: chacun, dans sa sphère de pouvoir, est souverain. Celui qui n'est souverain dans aucun domaine ne peut pas émettre de message, parce qu'il ne peut en recevoir, ni en recevoir, parce qu'il ne peut en émettre; il ne peut pas non plus les interpréter, parce qu'il est privé de tout moyen d'obtenir confirmation de la pertinence de ses interprétations. Tel serait le sort des hommes.

Il n'y a donc de message effectif que si, d'une manière ou d'une autre, la communication s'accompagne d'une perte de souveraineté - si l'émetteur, le messager et le destinataire ne sont pas libres dans l'acte de communication et perdent le droit de libre disposition dont ils jouissaient dans leur position initiale ${ }^{15}$.

Si l'émetteur n'est pas libre de communiquer un message dont il serait seul à connaître le sens, cela signifie qu'il doit lui donner un texte susceptible d'une réponse; si le messager doit transmettre le message tel quel, cela signifie qu'il est transmis comme texte pourvu d'un sens indépendamment de l'opération de transmission; si le destinataire doit le recevoir, cela signifie qu'il est censé y répondre. D'une façon ou d'une autre, le message, pour être un message, doit être objectivé afin de recevoir un mode d'existence qui lui permette d'être pour lui-même et qui,

une nouvelle interprétation des textes mêmes à propose desquels les questions se posent - et ainsi indéfiniment, à moins qu'une autorité hiérarchique (au sens le plus littéral!) n'ait le pouvoir d'y mettre un terme par pure et simple décision.

${ }_{15}$ Il est intéressant de noter que la philosophie théologique médiévale a aussi été aux prises avec ce problème de la perte de souveraineté, qu'elle a abordé il est vrai sur une tout autre voie: celle d'un soupçon pesant sur la toute-puissance divine d'être en son instance première (potentia absoluta) arbitraire et indifférente - parce que précisément toute-puissance - il s'agissait de fonder ontologiquement une théorie de la connaissance qui permette de penser la création comme accessible à la raison, donc, dans une autre terminologie, comme un message clair dont l'auteur même serait lié à des règles. Ce soupçon, elle s'est efforcée de le lever en tentant d'établir que la volonté divine était liée par le principe de non-contradiction, ce qui rendait impossible qu'elle produise n'importe quoi et garantissait l'intelligibilité de la création (comme potentia ordinanta). En un certain sens dernier philosophe médiéval, Descartes ira - presque - jusqu'au bout, admettant que rien n'exclut que Dieu ait voulu que les contradictoires puissent «être ensemble», mais considérant que «notre esprit» n'est pas «capable» de le «comprendre», parce que, «fini», il n'a pas été «créé» pour «concevoir comme possibles [les choses] que Dieu aurait pu rendre possibles, mais qu'il a toutefois voulu rendre impossibles» (Lettre au Père Mesland du 2 mars 1644). Ainsi, par son acte de création, c'est un monde ouvert à la compréhension que Dieu a produit, mais s'autolimitant par là même (et nous retrouverons l'idée d'autolimitation plus bas, avec le concept d'autotranscendance).Voir l'analyse de la philosophie de Guillaume d'Occam chez André de Muralt, L'enjeu de la philosophie médiévale, Leiden/New York/Köln, E.J. Brill, 1993, p. 242 ss, 300 ss (toutes références qui m’ont aimablement été fournies par Denis Ramelet). 
ainsi et seulement ainsi, contraint l'émetteur et le destinataire à l'émettre et à le recevoir en le soustrayant au pouvoir de leur subjectivité.

Or, cette objectivation, qui s'impose à l'émetteur, au messager et au destinataire, peut bien avoir pour origine l'acte de volonté de chacune des parties; mais elle ne peut être assurée que par quelque chose qui, parce qu'extérieur au message, lui donne son autonomie. C'est peut-être un paradoxe que les messages ne peuvent être pour eux-mêmes - libérés de la subjectivité souveraine de chacune des parties - que dans la mesure où ils ne peuvent être messages qu'en s'inscrivant dans un espace qui les dépasse: l'espace du code $^{16}$.

S'ensuit un paradoxe corollaire. L'espace du code dépasse tout autant les acteurs, en ce qu'aucun ne doit pouvoir en disposer. Les contraintes qu'il fait peser sur l'émetteur, le destinataire et le messager, perte de souveraineté pour chacun d'entre eux, recomposent s'ils les acceptent une liberté qui leur devient également commune ${ }^{17}$. Dans le mythe biblique, c'est bien en jetant la confusion dans leur langue - leur code - que Dieu a empêché de construire la tour de Babel.

4.2. On peut rejoindre ici la lecture de Kafka que propose François Ost, mais avec une approche explicitement éthique, en s'inspirant de Paul Ricœur ${ }^{18}$. «Je» ne peut s'identifier comme lui-même qu'en s'appelant «je» dans la relation à un «tu»: relation en même temps d'identification et d'altérité qui permet la reconnaissance des deux sujets chacun pour lui-même en même temps que pour et par l'autre ${ }^{19}$. Mais cette relation bipolaire implique un «il/elle» d'abord dans la personne $\mathrm{du}$ «tu» absent, relation triadique qui, sous la figure du «tiers», s'étend alors à l'ensemble de tous les «tu» potentiels: à tous ceux qui, parlant la même langue, sont de ce fait aptes à se reconnaître.

«Le "il/elle" représente l'institution, dans la mesure où celle-ci englobe tous les locuteurs d'une même langue naturelle qui ne se connaissent pas et ne sont reliés entre eux que par la reconnaissance des règles communes qui distinguent une langue d'une autre. Or cette reconnaissance ne se limite pas à la seule adoption par tous des mêmes règles, mais comporte la confiance que chacun met dans la règle de sincérité sans laquelle l'échange linguistique serait impossible. ${ }^{20}$

Dans l'hypothèse de lecture de François Ost, la faillite de cette opération de triangulation et l'impasse consécutive ferme la voie vers l'institutionnel:

«[En atteste] l'échec de l'institutionnalisation de l'intersubjectivité au travers des différentes étapes de l'identité solipsiste, de l'altérité, de la pluralité et du "il" normatif»; le

${ }^{16}$ Dans le rêve et le lapsus, le code est biaisé; l'analyse - par les indices que fournissent les associations libres - ramène sur les traces du code original.

17 A moins d'en rester au stade d'une existence «solipsiste» (Ost, p. 342). Celle-ci peut consister dans la résignation d'une soumission; mais elle peut aussi déboucher sur le conflit de souverainetés pures, donc sans signification et sans fin - la négation de la communauté sociale - conflit dont personne n'est assuré de sortir vainqueur, personne (sauf celui qui dispose, par un droit dont il décide seul, exclusivement de la Parole pure) ne jouissant par nature de la toute-puissance.

18 Ost, p. 341 ss. Voir Paul Ricœur, notamment dans : «Ethique et morale», in Lectures 1, Paris, Le Seuil, 1991, p. 256 s.; «Qui est le sujet de droit?», in: Le Juste 1, Paris, Editions Esprit, 1995, p. 33.

19 Evidemment pas dans le sens juridique du terme (code civil, code pénal, etc.).

${ }^{20}$ Ricœur, Le Juste 1, cit., p. 35. 
normatif se lisant comme loi qui, «loin d'être l'ukase terrifiant d'un "tu" tyrannique comminé à un être incapable de protester de son "je", [...] se prête au jeu de l'universalisation de sorte que le "tu" qui la profère s'y soumet comme un autre, comme n'importe quel autre.» ${ }^{21}$

On peut considérer, du moins provisoirement, que ce qui est appelé l'institution équivaut à ce que j’ai désigné par le terme de code: le monde de références communes qui, dépassant les acteurs d'une relation, leur permet de la mettre en œuvre. Un concept que François Ost utilise mérite à ce titre d'être relevé: l'universalisation. Il signifie que la relation ne tire pas son sens des seules singularités de l'émetteur, du destinataire et du message, mais de la mise en rapport avec le code. C'est à ce point que les lois se substituent à la Loi: au lieu d'être Parole pure, elles dessinent la frontière entre le permis et l'interdit, et cela sous une forme générale et abstraite, c'est-à-dire accessible à un décodage rationnel qui permet de vérifier leur bien-fondé - c'est une autre manière de définir le concept d'universalisation.

Mais peut-on se satisfaire d'en être arrivé là? En effet, se pose alors la question de la force coercitive de ce code: dans quelle mesure prédétermine-t-il le sens concrètement visé par les acteurs? En remplissant de lois l'espace vide de la Loi, ne tombe-t-on pas de Charybde en Scylla? S'il est impossible de savoir ce que veut celle-ci, l'est-il moins de prévoir ce que voudront celles-là?

Certes, ce n'est pas le problème de Kafka. Du moins pas explicitement: il ne traite pas de théorie politique. Mais peu importe ici, où il ne s'agit pas de commenter Kafka, mais de réfléchir au régime de l'Autorité en prenant pré-texte d'histoires racontées par lui. Au demeurant, à lire tant de choses sur l'image du Père dans son œuvre - donc du surmoi -, il n'est pas interdit de continuer sur le thème d'un autre mode de fonctionnement de l'autorité que celui d'un surmoi qui ne serait qu'un «automate imbécile ${ }^{22}$.

\section{LE CODE JURIDIQUE}

5.1. La question de la force coercitive des codes vaut à l'évidence particulièrement pour le code juridique. J'entends par là l'ensemble des textes normatifs qui composent le droit: constitutions, $\operatorname{codes}^{23}$, lois, règlements, plus généralement tous les textes formulant une règle de droit, une norme ${ }^{24}$. Avant de continuer, je veux mettre en évidence quelques traits distinctifs.

Les textes normatifs s'imposent dans toute situation singulière qui tombe dans le champ d'application de la règle qu'ils contiennent; et ce n'est pas par hasard que c'est dans leur caractère impératif que la doctrine classique voit leur critère

21 Ost, p. 344 s.

22 Ost, p. 346.

${ }^{23}$ Cette fois au sens juridique.

${ }^{24}$ Sur le concept de texte normatif, cf. Pierre Moor, Pour une théorie micropolitique du droit, Paris, PUF, 2005, p. 177 ss. A vrai dire, il inclut les textes individuels des jugements; mais, dans la suite, et pour ne pas avoir à expliquer ici le processus complexe de la formation de la norme juridique, je le restreins ici aux règles juridiques générales et abstraites, selon la définition généralement reçue. 
distinctif. Le code juridique se présente donc avec une caractéristique marquante par rapport à d'autres codes: alors qu'en général ceux-ci offrent la possibilité de combinaisons infinies de sens selon la sélection arbitraire à laquelle procèdent ceux qui entrent en communication, celui-là combine, ordonne, prédétermine déjà, dans la généralité et l'abstraction de ses formulations, le sens des situations singulières, ainsi soustraites au libre choix des parties à une relation juridique concrète. En d'autres mots, l'universel y absorbe le singulier, récupérant pour lui une entière souveraineté.

Il semble toutefois, au moins, que la liberté singulière existe partout où le droit ne dit rien. Mais ce n'est qu'en apparence: le droit ne dit jamais rien, il n'est jamais muet. Son silence signifie seulement que les conséquences d'un comportement singulier qui seraient préjudiciables à un quelconque tiers ne donneront lieu à aucune compensation en sa faveur: ce qui est aussi une règle de droit. Et l'adoption d'un texte explicite contraire peut d'ailleurs la modifier en tout temps.

Cette faculté qu'a le code d'être modifié en montre en outre une caractéristique essentielle: celle d'une expansivité dont il décide lui-même - lui-même, parce que ce sont des règles juridiques qui déterminent par qui, selon quelles procédures et à quelles conditions des normes nouvelles sont valablement émises. En ce sens, on peut dire que le code se modifie.

Enfin, et même surtout, alors même que les normes juridiques ne prédétermineraient pas elles-mêmes ce qui est de droit, parce qu'elles n'ont pas la précision nécessaire, le pouvoir de détermination du sens juridique d'une relation concrète est néanmoins réglé souverainement par le droit: il est transféré au juge, habilité en dernière instance à exercer la fonction d'universalisation. Le juge dit le droit, et même plus: par le juge, le droit se ditt $^{25}$.

5.2. On peut à ce point redéfinir le concept d'institutionnalisation, entendu ici non plus comme l'objectivation du normatif, mais plus précisément comme l'ordonnancement des instances qui ont la compétence d'émettre ou de dire le normatif juridique - loi ou sentence. Ces règles, qui font partie intégrante du code comme tout autre texte normatif ${ }^{26}$, confèrent un pouvoir de décision souverain: elles instituent les instances dont les décisions doivent être reçues, du point de vue du code, comme définitives et incontestables.

Le système juridique est dès lors en même temps acte de souveraineté - du législateur et du juge - et code. Lorsque les textes normatifs universalisent la singularité en ne retenant d'elle que ce qu'elles prédéterminent dans la généralité et l'abstraction de leur formulation, l'acte de souveraineté fixe le code: émetteur et code se confondent. Lorsque l'opération d'universalisation est l'œuvre du juge, l'acte de souveraineté dit le code: ce sont le messager et le code qui se confondent. Dans les deux cas, le destinataire, qui est le justiciable (formule passive !), n'a rien à répondre, parce que le code relève de la seule maîtrise de celui qui l'émet ou transmet le message élaboré sur sa base. En revanche - et c'est un acquis du système -, une fois que le message lui est transmis, il sait au moins de quoi il

${ }^{25}$ «Et même surtout »: ces mots du début de l'alinéa signifient que cette situation, loin de constituer une exception, est ordinaire dans tout système juridique: Moor, p. 66 ss, 178 ss.

${ }^{26}$ Règles primaires, au sens de Hart. 
répond. Ainsi non seulement s'évanouit le thème de l'autoaccusation généralisée mais apparaît aussi la présomption d'innocence: il appartient au code d'établir de quoi on est coupable ${ }^{27}$. Mais il n'en reste pas moins que l'institutionnalisation du code laisse subsister une partie du problème.

Car s'il veut ce que le droit lui dit, et sortir de sa passivité, le justiciable n'a à sa disposition qu'une seule voie: élever, contre l'acte de souveraineté, une revendication de justice en en appelant à un autre code, indépendant du système juridique.

Cependant, le code de justice présente un défaut qui lui est inhérent: il n'a par lui-même aucune force coercitive, et les institutions n'ont aucune obligation juridique de répondre aux messages dont il permet la formulation; il n'y a entre les deux codes aucun rapport de subordination, ni même de concurrence directe mais nous verrons que le droit assure autrement des possibilités d'articulation. Et les tentatives de fondation d'un code du code juridique - d'une surcodification n'ont jamais abouti, quelle qu'ait été l'imagination déployée à donner figure à un droit naturel ou à un contrat social. La cause de ces échecs réside dans la fonction même du code de justice, qui implique la plus grande liberté dans la formation des discours qui s'y réfèrent: plus seraient restreintes les combinaisons possibles de sens qu'il permet, plus limitées seraient les potentialités critiques qu'il offre; à la limite, il s'identifierait au droit - identification qui est d'ailleurs l'une des caractéristiques des régimes totalitaires, et par laquelle ils confisquent la justice. $\mathrm{Du}$ point de vue de la liberté imaginative dans la combinaison de ses éléments, le code de la justice doit donc être plus proche du code linguistique que du juridique ${ }^{28}$.

5.3. Que, sous la figure de l'acte de souveraineté, la Loi demeure dans les lois, on peut le montrer aussi en empruntant un autre chemin que celui que nous venons de suivre: celui du fonctionnement interne du code.

Il y a une distance irréductible entre la singularité du monde réel et sa reconstruction dans le code juridique. Pour s'appliquer, le droit définit sous forme de notions générales et abstraites les «faits » dont il reconnaît la pertinence pour son application. Les faits réels (singuliers) sont «qualifiés », c'est-à-dire reconstruits de telle manière à correspondre aux notions composant le texte normatif; sous cette forme en quelque sorte transfigurée, ils deviennent accessibles aux opérations argumentatives qui, dans le langage du code, mèneront à la conclusion du processus. Ainsi, tel comportement constituera une faute, tel groupement boisé une forêt «au sens de la loi ». Un récit est construit, qui, de manière irréfragable, est présumé réel.

Ce qui est vrai pour les faits en général l'est pour toute réalité: ce l'est très particulièrement pour la singularité des individus, que le code construit pour son fonctionnement propre comme «sujets de droit».

Les individus sont donc dépouillés d'eux-mêmes. Ils n'apparaissent, dès que le code se saisit d'eux, qu'en tant que ce code les représente: représentés, ils vont figurer comme les personnages de l'histoire fictive. Et, dans les procédures instituées par le code juridique, on assiste d'ailleurs, de manière caractéristique, à la

\footnotetext{
${ }^{27}$ Melkevik, n. 5 ci-dessus.

28 Sur les discours de justice et leur rapport au droit, cf. Moor, p. 251 ss.
} 
disparition des noms propres: il n'y est plus question de $M$. Untel ou de $\mathbf{M}^{\text {me }}$ Untel, mais de la «partie demanderesse » ou «défenderesse», du «recourant», de l'«intimé », de l' «accusé », du «président de la Cour», du «procureur» ou de son «substitut», du «témoin», de l'«expert». Les espaces et les rites judiciaires marquent aussi cette substitution par les représentations codées qui se mettent à la place du monde réel, qui en tiennent lieu.

L'opération n'est pas nécessitée par le fonctionnement du code: elle en est la définition même. On peut l'appeler «universalisation», comme le fait, semble-til, François Ost, puisque le processus repose sur l'assomption de l'individuel/concret dans les dimensions du général/abstrait. Ce terme évoque cependant par trop l'éthique kantienne pour pouvoir être employé sans précaution: il donne l'apparence d'évacuer la question essentielle, qui est celle du pouvoir qui se manifeste ainsi - comme si elle disparaissait dans la catalyse du passage à l'universel. Le récit construit par le juge au moyen des éléments que lui fournit le code repose sur une appropriation des singularités du réel: le sens en sera celui qui paraîtra à l'autorité la combinaison la plus judicieuse de ces éléments. Dans cette opération, les singularités seront absorbées par l'«universalisation », ce qui n'est pas autre chose que leur négation en tant que telles.

Et ce récit se termine par la sentence: «Tu es coupable», «tu es responsable», etc. Cette sentence, devenue définitive, c'est-à-dire rendue par la dernière instance compétente, est incontestable; elle ne peut qu'être acceptée telle quelle par les parties. Certes, elle peut être critiquée; mais, quel que soit l'éventuel vice dont on lui ferait grief (d'être injuste, et même d'être illégale ${ }^{29}$ ), elle va rester valable - sauf à la renverser par la force, ce qui, niant le code dans son entier, revient à se mettre hors-la-loi ou à provoquer une révolution. On voit par là, soit dit en passant, que l'impérativité dont on dit souvent qu'elle est la caractéristique définitoire des normes juridiques ne découle pas des textes normatifs qui servent de référence, mais du code lui-même.

Tel est la rançon à payer pour que le code juridique fonctionne. Certes, en compensation, il donne à l'équilibre des comportements singuliers un univers de signification autre que celui qui découlerait de simples rapports de pur fait; il ouvre ainsi à la communication. Il n'en demeure pas moins que ce qu'il accorde d'un côté, il est obligé de le reprendre de l'autre, et que ce que les justiciables reçoivent d'un côté, ils doivent le rétrocéder de l'autre.

La Loi réapparaît là même où on aurait espérer que les lois la fassent s'évanouir. Il reste, encore et toujours, une dette à payer mais sans possibilité d'une exécution libératrice parce que non définie, un message auquel toute réponse est déclarée irrecevable: le pouvoir de cette Loi-ci, aussi, se manifeste par une parole en elle-même définitive, «définitiveté» dont elle a le monopole.

\section{LE TRAVAIL DU DROIT}

6.1. Mais quand même: le code juridique ramène la Loi sur terre et, l'intégrant, lui superpose l'édifice des lois. Dans son champ d'application, il réussit à

\footnotetext{
${ }^{29}$ Sous réserve de motifs tout à fait exceptionnels, dits de révision, dans le jargon juridique.
} 
constituer une sorte d'autotranscendance: la Loi ne se manifeste que dans le cadre que lui impose l'ensemble des textes normatifs, qui tirent leur qualité - donc leur efficace signifiante - de leur appartenance au code juridique, aux conditions que ce dernier fixe lui-même. Un système de valeurs est ainsi formé dont les significations sont impératives par elles-mêmes pour tout sujet de droit - donc pour tout individu - par analogie, dira-t-on, elles sont transcendantes. Mais ce système est créé selon des compétences, des formes, des procédures intégrant, dans les Etats dits de droit, l'ensemble politiquement organisé des sujets de droit - d'où l'adjonction du suffixe. C'est bien dans cette autotranscendance que se réalise la transgression de la Loi, qui n'est plus reconnue que dans la figure des lois.

Cependant, nous ne sommes pas en présence d'un donné qui se manifesterait dans la révélation d'un quelconque fondement dont il suffirait de prendre (enfin!) conscience, ni même l'acquis inébranlable d'une évolution historique. Bien au contraire: c'est seulement par un travail constant que cet apprivoisement de la Loi est produit. L'objet de ce travail porte sur les textes normatifs, et il exige l'ouverture du code aux discours singuliers qui se réclament d'autres codes, dont le plus important, celui de la justice. Ce n'est pas par hasard que, plus haut, apparaît plusieurs fois le verbe «devoir», et il est nécessaire de rappeler en conclusion ce qu'il implique.

Car il peut - ou non - y avoir des entrées dans le code juridique, suivant l'aménagement institutionnel qu'il donne à la maîtrise qui s'exerce sur ce qu'il sert à manifester: et, par «entrée», j'entends des procédures et des institutions prévues ou assurées par le code lui-même pour se maintenir accessible aux messages qui lui sont adressés et se contraindre à y répondre. Pour le montrer, je distinguerai les plans macro- et micropolitique: d'abord celui de l'élaboration et de l'adoption des textes normatifs, ensuite celui de leur maniement (ce qu'on appelle l'«application»).

6.2. Premièrement, donc, une entrée macropolitique. L'adoption des textes normatifs peut suivre, en effet, deux modèles. A un extrême, celui d'un modèle clos, elle peut relever exclusivement d'une seule et même autorité, dont les actes sont sans appel. Cela signifie que celle-ci a la maîtrise exclusive de l'éaboration du contenu du code et qu'elle monopolise les communications faites sur la base du code de justice, n'ayant pas à répondre aux messages que les justiciables voudraient lui communiquer. Ce ne serait peut-être pas explicitement la Loi, mais ce serait l'Autorité - ce qui revient en fait au même.

A l'autre extrême, le modèle ouvert: celui dans lequel les discours de justice circulent librement et où existent des procédures dans lesquelles le législateur peut être appelé à rendre compte de ses actes. Il y a, plus ou moins régulièrement, confrontation entre le code juridique et celui de la justice. Ce modèle est celui de l'autonomie politique. Celle-ci est non seulement une autonomie sociale - celle qui se manifeste dans des procédures collectives juridiquement organisées; mais elle est aussi l'autonomie personnelle requise pour l'élaboration et la communication des discours de justice.

Cette double autonomie se concentre dans la figure du sujet de droit, qui, transcendant l'individu, le fait échapper aux contingences du réel en le dotant d'un statut codifié sous forme d'un ensemble indéterminé de droits. Cette indétermination n'est pas tellement celle du nombre de droits: c'est celle de la libre 
détermination du contenu que le sujet de droit va leur donner dans leur exercice concret $^{30}$. Et, comme ils appartiennent à tout sujet de droit, figure abstraite du code, cette liberté implique nécessairement la réciprocité des statuts dans les rapports interindividuels; le code juridique est ainsi un code des échanges dans la mesure où son aménagement institutionnel garantit la double autonomie.

Mais cette garantie ne vaut que dans la mesure où le monde réel en permet l'effectivité: les conditions sociales et économiques dans lesquelles les individus exercent en fait leur autonomie. Il doit y avoir ici suffisamment de droit (et de droits) à exercer pour que celle-ci ne soit pas simplement une figure du code: donc un travail de politique du droit, ce qui, par l'attention à porter à l'adéquation du code au monde réel, signifie un droit politique.

6.3. Mais il subsiste une sphère d'Autorité: celle de l'application du droit aux situations singulières, dans ce que j'appelle la dimension micropolitique. Car le libre exercice des droits se fait toujours sous réserve de leur reconnaissance par le code juridique en tant que droits. Les individus ne participent pas comme tels du code. Certes, ils peuvent parler la langue du code - ils disent qu'ils concluent un contrat, qu'ils sont innocents, etc. : mais ce ne sont que des propositions de droit, tant qu'une institution du code juridique - le juge - ne les reconnaît pas comme étant le droit ${ }^{31}$.

Se pose dès lors ici la question du pouvoir du juge: il a le monopole de la parole du code. Le juge est la «bouche de la loi», disait Montesquieu, qui voulait dire par là que l'institution judiciaire est soumise à l'empire de la loi, qu'elle a pour seule fonction de faire parler (du moins dans un régime de séparation des pouvoirs). Mais, comme elle en a seule la maîtrise - la compétence, dit le jargon juridique -, son pouvoir est, contrairement à ce que pensait Montesquieu, précisément à l'opposé d'être «nul »: dire le droit est la fonction exclusive du juge.

Ce monopole est un véritable pouvoir en ce que la loi n'a pas d'autre porteparole que le juge, ce qui place celui-ci dans la position d'être seul compétent pour certifier que ce qu'il dit sur la base du code est effectivement conforme au code. Et ce pouvoir est d'autant plus grand que la sphère d'autonomie que lui confèrent les textes normatifs est bien plus large qu'on le croit. Rares sont ceux dont la précision est telle que le juge, en les appliquant concrètement, ne fait qu'en répéter le contenu. Dans la plupart des cas, la densité normative des lois, c'est-à-dire le degré dans lequel elles prédéterminent exactement leur concrétisation, est faible, beaucoup plus faible que ce qu' on veut (faire) croire communément. En effet, une partie importante des textes normatifs, et fréquemment des textes normatifs les plus importants, ne prennent sens qu'au moment de leur détermination concrète, dans une situation individuelle: c'est seulement au moment même où ils sont appliqués par le juge qu'on peut connaître leur message ${ }^{32}$. Bien que circonscrite

${ }^{30}$ Sur l'articulation entre autonomie politique et individuelle, cf. Melkevik, p. 25 ss, 66; Moor, p. 226 ss.

31 Les individus, dans leurs rapports réciproques, font donc des anticipations sur leur signification juridique, anticipations qui, le plus souvent, n'ont pas à être vérifiées tant qu'aucun litige ne se produit; Moor, p. 196 ss.

32 Voir le développement sur les notions juridiques indéterminées, Moor, p. 66 ss, 179 ss. 
par les possibilités ouvertes par les procédures macropolitiques de déterminer dans les lois les significations impératives du code (l'autotranscendance), la Loi se retrouve ici dans le face-à-face du juge et des justiciables, certes à un niveau inférieur, déplacée, replacée, apprivoisée par le code, mais néanmoins bien réelle dans les fictions qu'elle permet de mettre en scène.

Peut-on pousser plus loin l'apprivoisement? Devant le juge, une procédure ouverte, du type démocratique que je viens de décrire, est inconcevable; ou plutôt, elle irait à l'encontre du code juridique. Celui-ci, en effet, postule que tout message doit se référer au code: cela signifie que toute décision concrète doit être motivée rationnellement sur la base du répertoire d'arguments fondés sur les textes normatifs. Or, une décision politique - et c'est bien d'une telle décision qu'il s'agirait, puisque la question serait de confronter l'arrêt du juge avec le code de la justice - sortirait du cadre ainsi délimité et annihilerait la garantie juridique qu'elle prétendrait fournir par l'acte même qui viserait à la fournir. Le modèle de la clôture s'impose donc. Par conséquent, ce n'est qu'à l'intérieur même du système juridique qu'on peut prévoir une circulation suffisante des messages pour en assurer l'éventuelle correction: publication de la jurisprudence, par exemple. En d'autres mots, la lecture des textes normatifs ne doit pas appartenir en monopole aux juges, mais être accessible à d'autres catégories de «lecteurs» dont la position leur permet d'être critiques de manière compétente ${ }^{33}$ : avocats présentant au juge les diverses interprétions possibles du code, professeurs de droit analysant, approuvant, réfutant la jurisprudence. L'appareil judiciaire lui-même est institutionnalisé de manière à permettre la critique interne: étagement des instances ouvrant les possibilités de recours, délibérations collégiales des tribunaux (surtout les cours supérieures, celles dont la jurisprudence fait autorité), publication des arrêts. Au-delà de ces possibilités de critiques internes, le sujet de droit en est réduit aux moyens macropolitiques, qui ne sont guère utiles au niveau individuel (sauf exception, ainsi si la personne en en question est aussi chef de gouvernement!).

Ici aussi, c'est un travail qui doit être accompli, un travail sur la production de sens en quoi consiste l'application du droit. Il est sans doute inévitable, comme pour tout travail, qu'il y ait des techniques propres, dont la maîtrise exige apprentissage. D'où une corporation, celle des juristes, en même temps et l'un par l'autre prêtres de la Loi et artisans des lois, corporation à laquelle est délégué le travail du code: une forme - à nouveau - apprivoisée de dépossession. Pourrait-il en être autrement, c'est vraisemblablement le rêve d'une utopie de la transparence totale - rêve qui, là où il devient réalité, est jungle (il n'y a aucun code) ou totalitarisme (tout est codifié).

Dura lex, sed lex.

Université de Lausanne

${ }^{33}$ Sur ce concept de «lecteur» des textes normatifs, cf. Moor, p. 217 ss. 